\title{
Single-cell genomics: coming of age
}

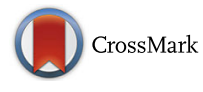

\author{
Sten Linnarsson ${ }^{1 *}$ and Sarah A. Teichmann ${ }^{2^{*}}$
}

Single-cell genomics is the study of the individuality of cells using omics approaches. Although young, the field has now entered its teenage years and is beginning to show clear signs of maturity. Its origins can be traced back to pioneering experiments that allowed the detection of gene expression in single cells by microarrays (reviewed in [1]). However, it was with the emergence of "next-generation" DNA sequencing that single-cell genomics really took off [2-4]. Although initial experiments were modest in size and resulted in noisy and incomplete data, they immediately revealed the great potential for biological discoveries. It soon became clear that the substantial technical and biological variability required data from many single cells in order to allow meaningful data mining and interpretation of the data [5]. Thus, the following years were spent pursuing a few lines of development: improvements in the accuracy and scope of single-cell methods and increasing throughput and reducing cost. Today, we are in a position to routinely measure gene expression in tens of thousands of single cells with high accuracy in terms of quantification of gene expression (albeit sensitivity in terms of detection of mRNAs varies significantly depending on protocol and sequencing depth). The costs are at least manageable and continue to decrease.

While single-cell RNA-seq is now mature and almost routine, technological development has shifted to other modalities: DNA, protein, chromatin modifications, and more. Single-cell whole-genome DNA sequencing is challenging because loss of material causes dropouts in the sequence and because sequencing errors are difficult to distinguish from real mutations. Despite these challenges, single human cortical neurons have been used to reconstruct lineages based on somatic mutations that had accumulated during development [6]. Similarly, clonal evolution within solid tumors can be revealed by detecting somatic copy number variations in single cells (reviewed in [7]).

\footnotetext{
* Correspondence: sten.linnarsson@ki.se; st9@sanger.ac.uk

'Department of Medical Biochemistry and Biophysics, Karolinska Institutet, Unit of Molecular Neurobiology, Scheeles väg 1, 17177 Stockholm, Sweden ${ }^{2}$ Wellcome Trust Sanger Institute, Wellcome Genome Campus, Hinxton CB10 1SD, UK
}

Another trend is the extension of single-cell analysis to measure epigenetic states such as DNA accessibility [8-10], methylation [11], and chromosome conformation [12]. Generally these methods pose similar challenges to DNA sequencing but offer access to pure cellular epigenetic states that are simply inaccessible by bulk methods.

Single-cell protein analysis occupies a different niche, where smaller numbers of proteins can be analyzed but in very large numbers of cells, classically using fluorescenceactivated cell sorting (FACS) for up to eight targets but more recently with mass cytometry targeting up to hundreds of proteins [13]. A limiting factor for protein analysis remains the requirement for high-quality affinity reagents such as antibodies.

Finally, a recent development (but see [14]) is the comination of methods to simultaneously measure two or modalities in single cells. For example, genome and nscriptome [15, 16], transcriptome and methylome [17, 18], and RNA and protein [19]. In the near future, single cells evolving in tumors to their genotypes.

Due to the speed with which single-cell genomics technologies are evolving, computational analysis methods are racing to keep up. Statistical and computational methods are at the heart of single-cell genomics and are critical to extracting meaningful information and biology from the data. Much work has focused on transcriptomic data analysis (e.g., reviewed in [20]) and in this special issue of Genome Biology there are examples of areas that benefit from bespoke computational approaches at the levels of both cells and genes. In terms of individual genes, a method to define significant differences in the cell-to-cell variation in gene expression (as opposed to mean expression levels) is reported [21] and one paper addresses expression states of long noncoding NAs [22]. In terms of cell-to-cell variation at the DNA vel, there is clearly tremendous scope for computamal method innovation in the area of tumor heterogeneity, addressed by Beerenwinkel and colleagues [23], and Markowetz and Ross [24] in this issue. 


\section{Recent applications}

Single-cell RNA sequencing has had a profound impact on our understanding of neuronal and hematopoietic cell types, as well as the immune system. Examples of novel insights in immunity include a window on to an unexpected plethora of dendritic cells in mouse immunity [25] and new regulators and subpopulations of $\mathrm{CD}^{4+}$ $\mathrm{T}$ cells [26-28]. In hematopoiesis, much single-cell transcriptomics work has focused on hematopoetic stem cells and the single-cell perspective has provided resolution of proliferation phenotypes [29-31]. A broader view of early specification of hematopoietic cell types was recently provided by Paul et al. [32]. Mead and colleagues [33] provide new insights into the erythroidmyeloid decision in this special issue.

While these publications all focus on mouse as a model, the unbiased nature of single-cell RNA sequencing provides great discovery potential in less-wellstudied animals. An example of this is the profiling of platelets (thrombocytes) from hematopoietic stem cells in zebrafish by Macaulay et al. [34]. In this issue, Pearson and Molinaro profile single cells in planarian regeneration [35]. Looking to the future, this type of approach can be expanded to comparative studies of many organisms across the animal kingdom in order to gain insight into the evolution of cell types.

The applicability of single-cell transcriptomics to nonadherent cells, such as those of hematopoiesis and immunity, is perhaps not surprising: these cells naturally exist as individual cells and remain stable after singlecell capture by FACS or in microfluidic devices. In the area of neurobiology and neuronal cell populations, the success of single-cell RNA sequencing is more surprising as these cells are bound up within networks of adherent junctions. Recently, comprehensive maps of cell types and subtypes have been produced for a number of key brain regions, including developing and adult cerebral cortex, and the day will come when we will have a full catalog of molecularly defined cell types in the whole nervous system. A particularly appealing application of such a reference atlas is in the use of human cerebral organoids to model human brain (which is otherwise inaccessible) in development and disease [36]. The fact that novel cell states, cell populations, and factors have been validated in this domain bodes well for a broader remit of single-cell transcriptomics to solid organs and tissues.

The DNA dimension, i.e., tracking mutations, copy number variations, and chromosomal aberrations at the single-cell level, has been important in both somatic cell populations such as neurons, as well as in cancer. In this issue, Park and colleagues show how single-cell dissection of tumor heterogeneity can translate directly into new combinatorial therapies in a xenograft model [37].

\section{Future prospects}

Gazing into our crystal ball, it is easy to predict an everincreasing role for single-cell genomics in discovery science, translational applications, and even ecology. The major driver of the single-cell genomics revolution is the step change in resolution of DNA and epigenetic and RNA sequencing down to the level of an individual cell. Since the cell is the basic building block of an organism, sequencing each cell in isolation provides information that is fundamentally different from genomic data that relates to ensembles of cells.

In terms of single-cell transcriptomics, the RNA content of a cell is deeply informative about its phenotype and function. This technique is so powerful and informative that it is likely that the community will ultimately map all mammalian organs, tissues, and cell types at single-cell resolution. A comprehensive resource such as this, effectively a "human cell atlas", would be a tremendously useful and unique reference data set for biology and medicine.

Like many previous waves of biotechnology, single-cell genomics started in academia and basic research but is now set to move into pharma and the clinic. Once an atlas of human cell types is available, any diseased tissue can be compared with it. Cancer, in particular, the prototypical single-cell disease, will be particularly apt for a single-cell analysis overhaul. Diagnostic assays, which are currently based on crude bulk methods, will be tremendously more powerful once they are brought down to the level of the individual transformed cell, in the context of its surrounding tissue, with cell-type specificity and a full understanding of somatic mutations.

We are excited to be part of a community that has already achieved a lot, as showcased in this special issue, yet clearly still has a long and interesting journey ahead of it.

\section{Abbreviation \\ FACS: fluorescence-activated cell sorting.}

Competing interests

The authors declare that they have no competing interests.

Authors' contributions

Both authors read and approved the final manuscript.

Published online: 10 May 2016

\footnotetext{
References

1. Tang F, Lao K, Surani MA. Development and applications of single-cell transcriptome analysis. Nat Methods. 2011;8:S6-11.

2. Tang F, Barbacioru C, Wang Y, Nordman E, Lee C, Xu N, et al. mRNA-Seq whole-transcriptome analysis of a single cell. Nat Methods. 2009;6:377-82.

3. Islam S, Kjallquist U, Moliner A, Zajac P, Fan JB, Lonnerberg P, et al. Characterization of the single-cell transcriptional landscape by highly multiplex RNA-seq. Genome Res. 2011:21:1160-7.

4. Ramskold D, Luo S, Wang YC, Li R, Deng Q, Faridani OR, et al. Full-length mRNA-seq from single-cell levels of RNA and individual circulating tumor cells. Nat Biotechnol. 2012;30:777-82.
} 
5. Grun D, van Oudenaarden A. Design and analysis of single-cell sequencing experiments. Cell. 2015;163:799-810.

6. Lodato MA, Woodworth MB, Lee S, Evrony GD, Mehta BK, Karger A, et al. Somatic mutation in single human neurons tracks developmental and transcriptional history. Science. 2015;350:94-8.

7. Navin NE. The first five years of single-cell cancer genomics and beyond. Genome Res. 2015;25:1499-507.

8. Buenrostro JD, Wu B, Litzenburger UM, Ruff D, Gonzales ML, Snyder MP, et al. Single-cell chromatin accessibility reveals principles of regulatory variation. Nature. 2015;523:486-90.

9. Cusanovich DA, Daza R, Adey A, Pliner HA, Christiansen L, Gunderson KL, et al. Epigenetics. Multiplex single-cell profiling of chromatin accessibility by combinatorial cellular indexing. Science. 2015;348:910-4.

10. Jin W, Tang Q, Wan M, Cui K, Zhang Y, Ren G, et al. Genome-wide detection of DNase I hypersensitive sites in single cells and FFPE tissue samples. Nature. 2015;528:142-6.

11. Smallwood SA, Lee HJ, Angermueller C, Krueger F, Saadeh H, Peat J, et al. Single-cell genome-wide bisulfite sequencing for assessing epigenetic heterogeneity. Nat Methods. 2014;11:817-20.

12. Nagano T, Lubling Y, Stevens TJ, Schoenfelder S, Yaffe E, Dean W, et al. Single-cell $\mathrm{Hi}$-C reveals cell-to-cell variability in chromosome structure. Nature. 2013;502:59-64.

13. Bendall SC, Simonds EF, Qiu P, Amir e-AD, Krutzik PO, Finck R, et al. Singlecell mass cytometry of differential immune and drug responses across a human hematopoietic continuum. Science. 2011;332:687-96.

14. Klein CA, Seidl S, Petat-Dutter K, Offner S, Geigl JB, Schmidt-Kittler O, et al. Combined transcriptome and genome analysis of single micrometastatic cells. Nat Biotechnol. 2002;20:387-92.

15. Macaulay IC, Haerty W, Kumar P, Li YI, Hu TX, Teng MJ, et al. G\&T-seq: parallel sequencing of single-cell genomes and transcriptomes. Nat Methods. 2015;12:519-22.

16. Dey SS, Kester L, Spanjaard B, Bienko M, van Oudenaarden A. Integrated genome and transcriptome sequencing of the same cell. Nat Biotechnol. 2015;33:285-9.

17. Moroz LL, Kohn AB. Single-neuron transcriptome and methylome sequencing for epigenomic analysis of aging. Methods Mol Biol. 2013;1048:323-52.

18. Angermueller C, Clark SJ, Lee HJ, Macaulay IC, Teng MJ, Hu TX, et al. Parallel single-cell sequencing links transcriptional and epigenetic heterogeneity. Nat Methods. 2016:13:229-32.

19. Darmanis S, Gallant CJ, Marinescu VD, Niklasson M, Segerman A, Flamourakis $\mathrm{G}$, et al. Simultaneous multiplexed measurement of RNA and proteins in single cells. Cell Rep. 2016;14:380-9.

20. Stegle O, Teichmann SA, Marioni JC. Computational and analytical challenges in single-cell transcriptomics. Nat Rev Genet. 2015;16:133-45.

21. Vallejos CA, Richardson S, Marioni JC. Beyond comparisons of means: understanding changes in gene expression at the single-cell level. Genome Biol. 2016;17:70.

22. Liu SJ, Nowakowski TJ, Pollen AA, Lui JH, Horlbeck MA, Attenello FJ, et al. Single-cell analysis of long non-coding RNAs in the developing human neocortex. Genome Biol. 2016:17:67.

23. Jahn K, Kuipers J, Beerenwinkel N. Tree inference for single-cell data. Genome Biol. 2016. doi:10.1186/s13059-016-0936-X.

24. Ross EM, Markowetz F. OncoNEM: inferring tumor evolution from single-cell sequencing data. Genome Biol. 2016;17:69.

25. Jaitin DA, Kenigsberg E, Keren-Shaul H, Elefant N, Paul F, Zaretsky I, et al. Massively parallel single-cell RNA-seq for marker-free decomposition of tissues into cell types. Science. 2014;343:776-9.

26. Mahata B, Zhang X, Kolodziejczyk AA, Proserpio V, Haim-Vilmovsky L, Taylor AE, et al. Single-cell RNA sequencing reveals Thelper cells synthesizing steroids de novo to contribute to immune homeostasis. Cell Rep. 2014;7:1130-42.

27. Proserpio V, Piccolo A, Haim-Vilmovsky L, Kar G, Lonnberg T, Svensson V, et al. Single cell analysis of CD4+ T cell differentiation reveals three major cell states and progressive acceleration of proliferation. Genome Biol. 2016. doi:10.1186/s13059-016-0957-5.

28. Gaublomme JT, Yosef N, Lee Y, Gertner RS, Yang LV, Wu C, et al. Single-cell genomics unveils critical regulators of Th17 cell pathogenicity. Cell. 2015; 163:1400-12.

29. Kowalczyk MS, Tirosh I, Heckl D, Rao TN, Dixit A, Haas BJ, et al. Single-cell RNA-seq reveals changes in cell cycle and differentiation programs upon aging of hematopoietic stem cells. Genome Res. 2015;25:1860-72.

30. Tsang JC, Yu Y, Burke S, Buettner F, Wang C, Kolodziejczyk AA, et al. Single-cell transcriptomic reconstruction reveals cell cycle and multi-lineage differentiation defects in Bcl11a-deficient hematopoietic stem cells. Genome Biol. 2015;16:178.

31. Wilson NK, Kent DG, Buettner F, Shehata M, Macaulay IC, Calero-Nieto FJ, et al. Combined single-cell functional and gene expression analysis resolves heterogeneity within stem cell populations. Cell Stem Cell. 2015;16:712-24.

32. Paul F, Arkin Y, Giladi A, Jaitin DA, Kenigsberg E, Keren-Shaul H, et al. Transcriptional heterogeneity and lineage commitment in myeloid progenitors. Cell. 2015;163:1663-77.

33. Psaila B, Barkas N, Iskander D, Roy A, Anderson S, Ashley N, et al. Single-cell profiling of human megakaryocyte-erythroid progenitors identifies distinct megakaryocyte and erythroid differentiation pathways. Genome Biol. 2016. doi:10.1186/s13059-016-0939-7.

34. Macaulay IC, Svensson V, Labalette C, Ferreira L, Hamey F, Voet T, et al. Single-cell RNA-sequencing reveals a continuous spectrum of differentiation in hematopoietic cells. Cell Rep. 2016;14:966-77.

35. Molinaro AM, Pearson BJ. In silico lineage tracing through single cell transcriptomics identifies a neural stem cell population in planarians. Genome Biol. 2016. doi:10.1186/s13059-016-0937-9.

36. Camp JG, Badsha F, Florio M, Kanton S, Gerber T, Wilsch-Brauninger M, et al. Human cerebral organoids recapitulate gene expression programs of fetal neocortex development. Proc Natl Acad Sci U S A. 2015;112:15672-7.

37. Kim KT, Lee HW, Lee HO, Song HJ, Jeong DE, Shin S, et al. Application of single-cell RNA-sequencing in optimizing a combinatorial therapeutic strategy in metastatic renal cell carcinoma. Genome Biol. 2016. doi:10.1186/ s13059-016-0945-9. 